\title{
Fostering Knowledge Mode Conversion in New Product Development Environment
}

\author{
Eduardo González ${ }^{1}$, David Guerra-Zubiaga ${ }^{1}$, and Manuel Contero ${ }^{2}$ \\ ${ }^{1}$ Institute of Technology and Higher Studies of Monterrey, Center of Innovation in Design \\ and Technology, Ave. Garza Sada 2501, 64849 Monterrey, Mexico \\ \{egm, david.guerra\} aitesm.mx \\ ${ }^{2}$ Polytechnic University of Valencia, School of Industrial Engineering, \\ Camino de Vera s/n, 46022 Valencia, Spain \\ mcontero@degi.upv.es
}

\begin{abstract}
The creation of new knowledge that comes from Knowledge Mode Conversion (KMC) activities improves New Product Development (NPD) activities. However, there is still limited understanding of "how" a Knowledge Mode Conversion activity varies using collaborative and conversational technologies to improve product development performance. The main contribution of this research is to establish a set of indicators that can be used as guides to help identify effective Knowledge Mode Conversion activities that can be useful for organizations whose performance rely upon effective new product development activities. These indicators are obtained evaluating and comparing documents stored in a Product Data Management System (PDM) for differing levels of semantic significance, applying Latent Semantic Analysis (LSA). This provides a linkage between New Knowledge creation and the development of Capabilities for KMC, and a better understanding of "how" design teams improve their performance.
\end{abstract}

Keywords: Knowledge Mode Conversion, Latent Semantic Analysis, Collaborative Engineering, Knowledge sharing, Product Design.

\section{Introduction}

Knowledge creation at New Product Development (NPD) process is a key strategy that helps enterprises to survive in a global and highly competitive market. As manufacturers realize that they must compete globally, they recognize that developing and launching better new products is a critical issue. While Product Lifecycle Management (PLM) applications are core to this initiative, success will be determined by how well manufacturers assess the most critical underlying processes and resources. According to a report [14] from "AMR Research" a consulting firm focused on supply chain and enterprise-wide manufacturing applications:

- $95 \%$ of new consumer products ('96-'01) lost money or broke even.

- Median new vehicle development costs are $\$ 500$ million higher than best in class.

- Poor NPD costs apparel makers $30 \%$ of annual revenue in markdowns.

- Food retailers spend $\$ 957 \mathrm{~K}$ per store on new products that fail. 
In dealing with the necessity to generate an effective method for the creation of new knowledge within NPD, it is needed to support the knowledge transfer capability[16].

This capability has been viewed as critical for competitive advantage [1]. It has been referred to as "integrative capability" [2] and a "core competence" [3] business.

This growth in importance of the NPD process points us towards the need to consider a way of monitoring this process, taking the Knowledge Creation derived from KMC activities as the main variable to track during the NPD stages.

This paper reports a research over several years conducted at ITESM University by undergraduate design teams. The paper provides indicators showing proper Virtual Collaborative monitoring and Conversational technologies supporting knowledge transfer and combined capabilities that result in an effective method for KMC.

The design process is considered a socio-technical activity, and the design team acts as the mechanism for knowledge mobilization. This research establish a way for measuring the ability of individuals involved in design project teams, to come together to create, share, maintain and combine their individual knowledge, and transform that knowledge into new knowledge $[4,12,17]$.

\section{Background}

A key issue in conceptualizing a KMC for the NPD would be the definition of what Nonaka described as "tacit" knowledge and the description of the process that transform "tacit" into the "explicit" or possibly organized knowledge [5]. According to Nonaka, knowledge is created through a continuous dialog between tacit and explicit knowledge, this concept implies that the flow of information between tacit and explicit knowledge foster the Knowledge Mode Conversion.

Explicit knowledge is defined as knowledge that can be transmitted in formal, systematic language, whereas tacit knowledge refers to knowledge that has an individual character and is therefore difficult to formalize and communicate [4]. Nonaka presented four modes of Knowledge Conversion between tacit and explicit:

- Socialization (tacit to tacit),

- Internalization (explicit to tacit),

- Externalization (tacit to explicit), and

- Combination (explicit to explicit).

Figure 1 shows the graphical representation of Nonaka's Knowledge Spiral. Socialization represents the interaction between individuals. Combination involves combining explicit knowledge through meeting and conversation or using information systems. Internalization converts explicit knowledge into tacit knowledge and externalization converts tacit knowledge into explicit knowledge.

Organizational knowledge creation takes place when all four modes of knowledge conversion form a continual cycle triggered by such actions as team interactions, dialogue, metaphors, coordination, documentation, experimentation, and learning by doing, etc. Organizational knowledge creation can be viewed as an upward spiral process from the individual level to the collective group level, and then to the organizational level, sometimes to the inter-organizational level. [9] 
Conversational knowledge creation emerged as a popular way for NPD to create knowledge in the context of KMC. With a collaborative environment, individuals share knowledge through dialog with questions and answers. KMC model of knowledge creation is different from other models, where knowledge is for instance created through abstraction or aggregation of information $[6,16]$.

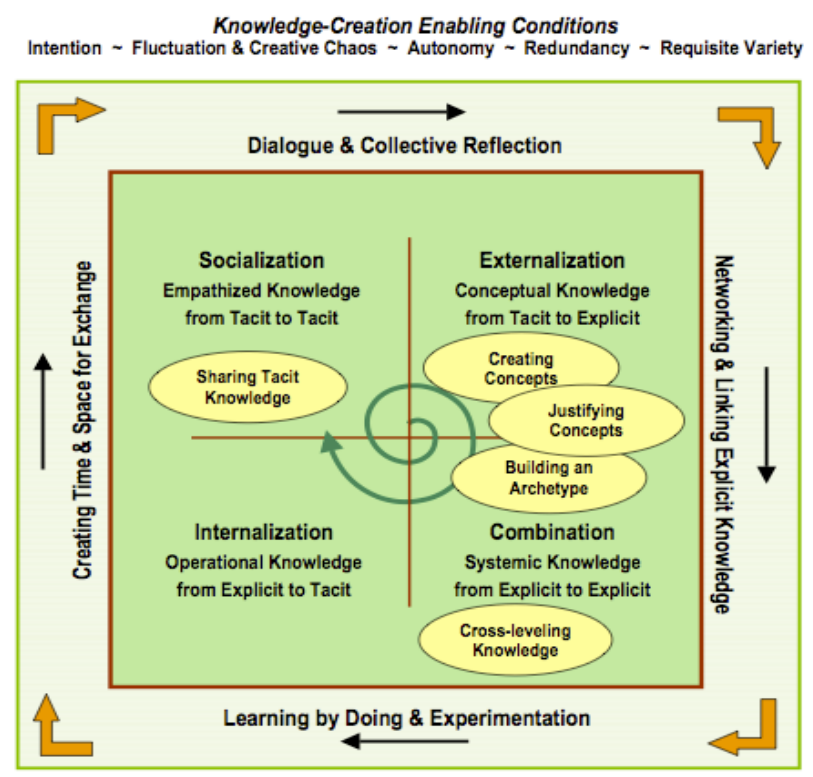

Fig. 1. A graphic depiction of Nonaka's \& Takeuchi's theory on knowledge creation [9]

There is evidence shown by Agogino, that shared understanding is produced in the NPD process. Semantic coherence concerning the meaning related to a written text, can be understood as a property of a set of related descriptions, but it can also be understood as a property of a single description with respect to a set of descriptions whose semantic coherence has been established, and we can also derivate the semantic coherence of a set of descriptions as determined by their pair-wise coherence. The coherence metric applied in this research is based on the cosine measurement, which is appropriate for measuring the pair-wise coherence of documents consecutive in time to detect the change in coherence over discrete periods of time $[15,8]$.

In the context of characterizing design team performance, semantic coherence levels have been measured in all stages of a design team process [7, 17]. The present research is based in the idea that, it is possible to measure the semantic coherence levels for documents identified as strongly associated with Knowledge Modes [9] and knowledge modeling [16]. 


\section{Research Method}

The general approach to this research involves the capture of documents generated within a design team, working on a collaborative project consisting in the creation of an unmanned air vehicle. The project involves the participation of more than 30 undergraduate students of different disciplines over a 3-year period that uses "Central Desktop" [7] as the Product Data Management (PDM) platform. According to the nature of the data contained in the documents of the PDM system, it was decided to use LSA as one of the methods of this research.

The main idea behind LSA for analyzing the cognitive processes underlying, modes of knowledge conversion on documents, is that by looking at the entire range of words chosen in a wide variety of texts; patterns will emerge in terms of word choice, as well as word and document meaning. LSA is a statistical technique for extracting and inferring relations of expected contextual usage of words in a given document. For this matter it was decided to perform a semantic analyses in the documents identified as typically used in the supporting technologies for each KMC described by Brohman [11] in Table 1.

Table 1. Modes of knowledge creation and supporting technologies

\begin{tabular}{|l|l|l|l|}
\hline \multicolumn{1}{|c|}{ Mode } & $\begin{array}{c}\text { Interplay } \\
\text { Relationship }\end{array}$ & \multicolumn{1}{|c|}{ Definition } & \multicolumn{1}{c|}{$\begin{array}{c}\text { Information } \\
\text { System }\end{array}$} \\
\hline Socialization & Tacit to Tacit & $\begin{array}{l}\text { Conversion of tacit knowledge to new tacit } \\
\text { knowledge through social interactions and shared } \\
\text { experience among organizational members. }\end{array}$ & $\begin{array}{l}\text { Computer-mediated } \\
\text { communication }\end{array}$ \\
\hline Combination & $\begin{array}{l}\text { Explicit to } \\
\text { Explicit }\end{array}$ & $\begin{array}{l}\text { Creation of new explicit knowledge by merging, } \\
\text { categorizing, reclassifying, and synthesizing } \\
\text { existing explicit knowledge. }\end{array}$ & $\begin{array}{l}\text { Data warehousing, data mining, } \\
\text { document repositories, and } \\
\text { software agents }\end{array}$ \\
\hline Externalization & Tacit to Explicit & $\begin{array}{l}\text { Creation of new explicit knowledge from tacit } \\
\text { knowledge by supporting beliefs, paradigms, and } \\
\text { view-points with codified evidence. }\end{array}$ & $\begin{array}{l}\text { Collaboration, coordination, \& } \\
\text { (e.g., GSS) }\end{array}$ \\
\hline Internalization & Explicit to Tacit & $\begin{array}{l}\text { Creation of new tacit knowledge from explicit } \\
\text { knowledge by understanding and learning from } \\
\text { reports or discussion. }\end{array}$ & Intranets \\
\hline
\end{tabular}

First thing to apply LSA is to present the text that is going to be analyzed as a matrix in which each row stands for a singular word and each column stands for a given text or other context. The intersections contain the frequency with which the word of its row appears in the given text denoted by its column. After that, the intersection values are weighted by a function that expresses both the word's importance in the particular portion of text and the degree to which the word type carries information in the domain of the general text. Then LSA applies statistical inferences to determine the semantic coherence [3]. We can observe from Figure 2 that LSA applies Singular Value Decomposition (SVD) to the matrix. In SVD, a rectangular matrix is decomposed into the product of three other matrices. One component matrix describes the original row entities as vectors of derived orthogonal factor values, another describes the original column entities in the same way, and the third is a diagonal matrix containing scaling values such that when the three components are matrix-multiplied, the original matrix is reconstructed.

It has been established three methods [3] to calculate coherence trough measuring shared semantic similarity between design team documentation. These measures can be obtained performing one of the following operations on the associated high dimensional vectorial representation of text and terms on a semantic space: 


\section{- Dot Product}

- Cosine

\section{- Euclidean}

$$
\begin{aligned}
& x \cdot y=\sum_{i=1}^{N} x_{i} y_{i} \\
& \cos \left(\theta_{x y}\right)=\frac{x \cdot y}{|x||y|}
\end{aligned}
$$

$\operatorname{euclid}(x, y)=\sqrt{\sum_{i=1}^{N}\left(x_{i}-y_{i}\right)^{2}}$

According to Landauer [3] a Semantic Space section, is a mathematical representation of a large body of text. Every term, every text, and every novel combinaion of terms has a high dimensional vector representation. When you compare two terms you compare the cosine of the angle between the vectors representing the terms.

The present research employed the cosine method to compare semantic similarities and used the "LSA@ CU Boulder" [13] web software application to perform the corresponding analysis (see Figure 3 for a screenshot of this application).

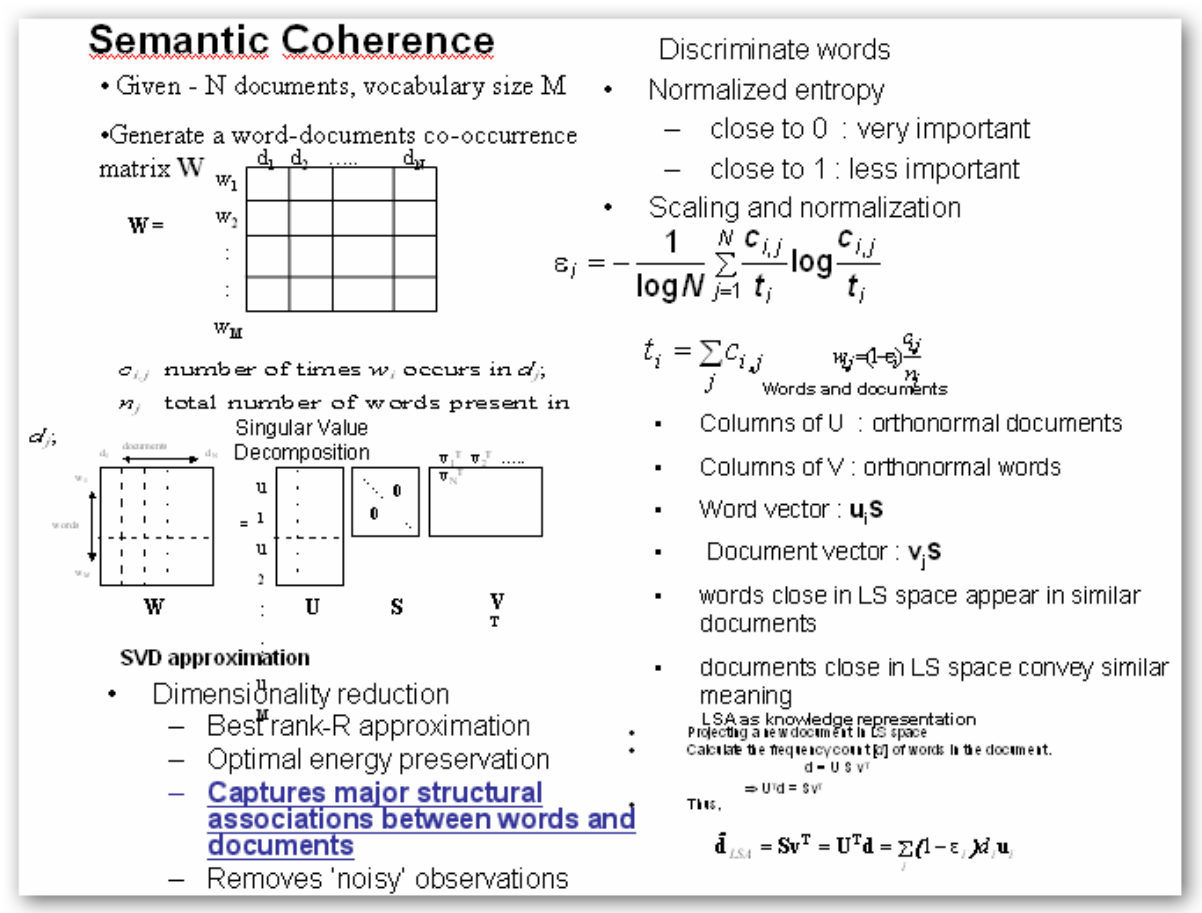

Fig. 2. Algorithmic structure of the LSA technique. Based on [4] and [8]. 


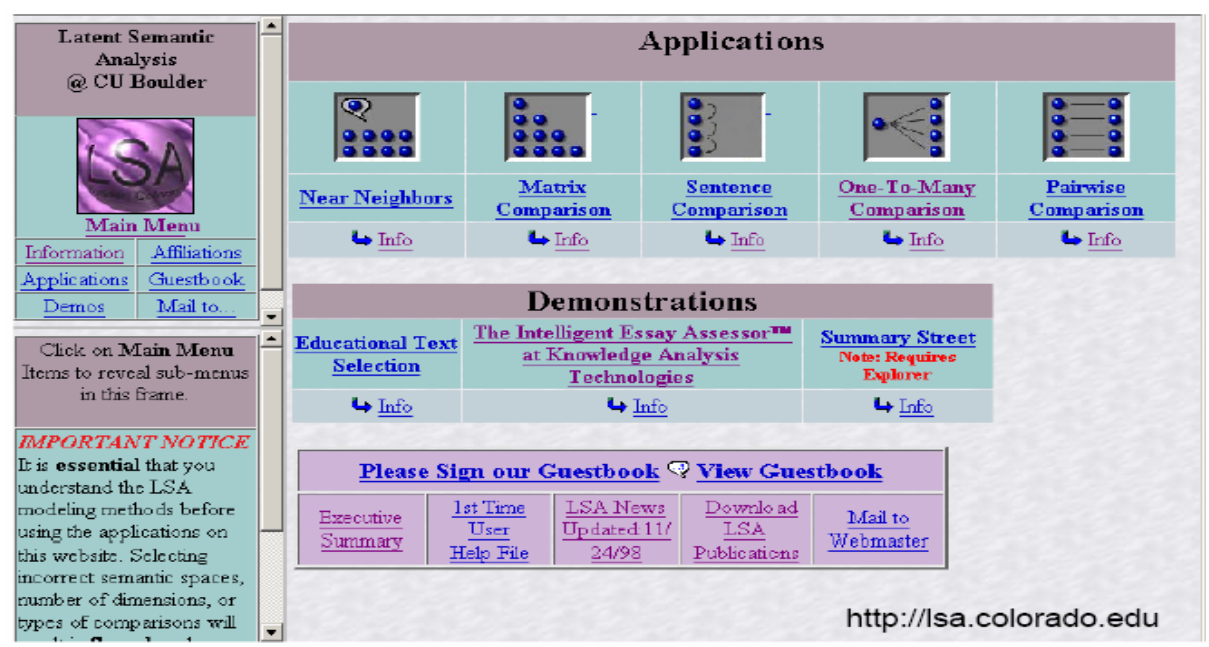

Fig. 3. Latent Semantic Analysis@ CU Boulder [13]

The following method was employed to measure semantic coherence:

1. Documents of each design team are grouped and categorized using the information system technologies identified as typically used for each KMC described in Table 1. For this matter the following criteria for categorization of documents had been used:

- Documents in the Socialization Knowledge Mode are those that are elaborated as a result of team activities of shared experience.

In our case: All documents related with how students learned from mentors or professors through a process of observation and repetition. In the building of the unmanned aerial vehicle that required new processes and their implementation. Each team developed documents related to the new work practices and routines to integrate and make sense of the NPD.

- Documents in the Combination Knowledge Mode are those that are elaborated as a result of a process by which explicit knowledge held by individuals is shared.

In our case: All documents related with how students interacted around the process of designing the unmanned air vehicle. Students had to combine knowledge about how best to integrate the aerial vehicle during formal or informal meetings.

- Documents in the Internalization Knowledge Mode are those that are elaborated as a result of the traditional concept of learning process. However, because in this case explicit knowledge is converted to tacit, it is the process or action that enables the conversion. In the case of a new technology tool, the act of using the tool enables the internalization of knowledge.

In our case: All documents related with how students improve their knowledge and skills of new hardware and software 
- Documents in the Externalization Knowledge Mode as an opposite case from Internalization are those that are elaborated as a result of the conversion of tacit knowledge to explicit knowledge. Because tacit knowledge is not definable directly in language, metaphors are often used to explain the knowledge concept.

In our case: All documents related with how individual students describe to the team how he or she uses their new knowledge and skills of new hardware and software to better perform in the team activities.

2. Calculate the semantic coherence of each team's documentation, according to LSA@CU Boulder software.

3. Compute graphical data of the semantic coherence of each team's documentation versus design stages.

\section{Results}

In order to characterize the design team performance, documents identified as typically used in a Knowledge Mode, as described in the previous point, were analyzed to obtain a measure of semantic coherence using LSA. This not only gave us a measure trough all stages of the NPD, but also gave us the opportunity to zoom-in the Knowledge Creation Process proponed by Nonaka.

Text from documents was analyzed using the "Sentence Comparison Method Interface" of LSA @ CU Boulder. The interface allows you to compare the similarity of sequential sentences within a particular LSA space. Each sentence is compared to next sentence. The program will automatically parse the input into sentences [13].

The result of the application of this interface is a measure of the semantic coherence within the text analyzed. The measures of coherence for all documents used by the design teams in all the design stages were collected and used to compute graphical data.

The data of semantic coherence between three design teams was compared not only taking in a count the design stages, but also the Knowledge Modes described by Nonaka.

A key result from this study is the determination of a method for collaborativebased environments that identifies which product development activities promote the growth of coherence levels in knowledge modes. This method facilitates to monitor activities that can be indicators of semantic significance that promote KMC. The presence of increasing significance, in KMC, during the design process is desirable.

In the other hand, decreasing in semantic significance in product development activities denote disruption and disfunctionality as already observed by Agonino [7].

The difference between this research and other previously released is that with this new proposed approach it is possible to track coherence measures within the Knowledge Modes. In this way, design teams could monitor, in a collaborative-based environment, which product development activities within its supporting technologies promote higher semantic coherence levels. 
Table 2. Team data of coherence media for Knowledge Modes

\begin{tabular}{|c|c|c|c|c|c|c|c|c|c|c|c|c|c|}
\hline week & TA Soc & TA Ext & Comb & Intern & B Soc & TB Ext & Comb & 3 Intern & C Soc & C Ext & Comb & Intern & \\
\hline$w 1$ & 0.1 & 0.31 & 0.32 & -0.15 & -0.14 & -0.13 & -0.16 & -0.15 & 0.14 & -0.11 & 0.2 & 0.15 & DESIGN STAGES \\
\hline$w 2$ & -0.22 & 0.33 & 0.35 & -0.1 & -0.22 & 0.33 & -0.12 & -0.18 & -0.13 & 0.22 & 0.25 & -0.21 & \\
\hline w3 & 0.31 & 0.36 & 0.54 & 0.13 & -0.1 & -0.12 & 0.45 & -0.32 & 0.22 & 0.12 & 0.33 & 0.14 & \\
\hline w5 & 0.33 & 0.44 & 0.62 & -0.22 & 0.16 & 0.34 & 0.62 & -0.12 & 0.33 & 0.44 & 0.32 & 0.32 & \\
\hline$w 6$ & 0.33 & 0.56 & 0.54 & 0.26 & 0.23 & 0.56 & 0.45 & 0.46 & 0.23 & 0.66 & -0.14 & -0.23 & Concept Design \\
\hline$w 7$ & 0.3 & 0.22 & 0.67 & 0.22 & 0.33 & 0.82 & 0.34 & 0.39 & 0.23 & 0.62 & 0.23 & 0.31 & \\
\hline$w 10$ & 0.31 & 0.92 & 0.34 & -0.25 & 0.51 & 0.72 & 0.77 & 0.25 & 0.41 & 0.88 & 0.43 & 0.65 & Preliminary Design \\
\hline$w 11$ & 0.6 & 0.92 & 0.89 & 0.45 & 0.66 & 0.52 & 0.89 & 0.55 & 0.45 & 0.9 & 0.66 & 0.75 & \\
\hline$w 12$ & 0.24 & 0.95 & 0.95 & 0.54 & 0.27 & 0.88 & 0.77 & 0.39 & 0.74 & 0.89 & 0.85 & 0.43 & \\
\hline$w 13$ & 0.66 & 0.87 & 0.88 & 0.48 & 0.56 & 0.66 & 0.73 & 0.5 & 0.66 & 0.87 & 0.76 & 0.81 & \\
\hline$w 14$ & 0.54 & 0.91 & 0.88 & 0.33 & 0.49 & 0.9 & 0.77 & 0.63 & 0.78 & 0.81 & 0.85 & 0.83 & Final Design \\
\hline
\end{tabular}

In our case, we anticipated through observing Socialization Knowledge Mode coherence measures of design teams (as seen on Table 2), the need to trigger interaction activities where our students could learn from observation and imitation of a professor, tutor or instructor. For this matter a manufacturing facilities was created where a professional instructor constructed with our students special parts for the aerial vehicle, with the purpose of facilitating this kind of knowledge sharing.

In the other hand, it was also promoted activities to obtain a collective reflection on how the new skills and abilities related to the use of new hardware and software tools, were improving each individual performance toward the improvement of the performance of the whole team. These collective reflection activities were inducing growing in coherence for both Externalization and Internalization Knowledge Mode, but for this last Mode, activities of hands-on learning by doing of the new hardware and software tools were also implemented.

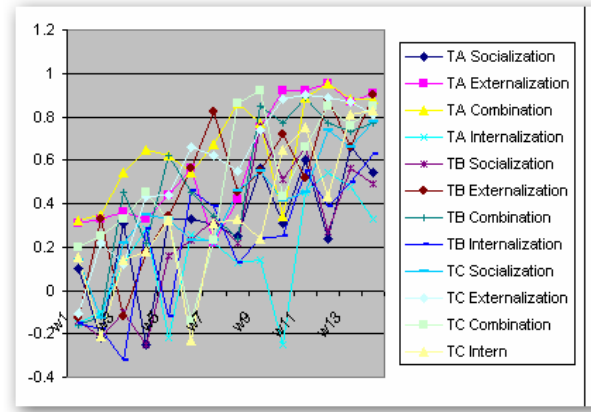

SEMANTIC COHERENCE FOR TEAMS A,B \& C

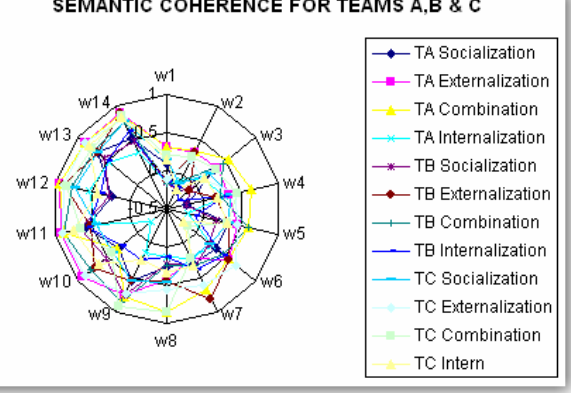

Fig. 4. Coherence variation in time of Knowledge Mode level

Activities with the intention to grow coherence measures for the Combination Knowledge Mode were deployed with the purpose of coordinate the work of the different sections within a design team. These activities considered the way teams were connecting existing or previous knowledge or data with other explicit knowledge in the project of the NPD. 
In our research, was observed that coherence levels grow over each design stage, but also was observed a correlation between the growing in coherence in the Socialization Mode and the grow of coherence in the Externalization Mode, but with a slight delay in time (see Figure 4 and Table 2).

So once this sequence was observed work was focused on measuring the correlation between coherence grow in a particular Knowledge Mode and the growing of the coherence in the next and sequential Knowledge Mode.

\section{Conclusions}

This paper has surveyed the following activities that can trigger Knowledge Mode Conversion in the processes underlying organizational knowledge creation using the Nonaka model and that in an empirical way were detected to improve the semantic coherence measures for the Knowledge Mode:

- Activities to learn from observation and imitation of a professor, tutor or instructor.

- Activities to obtain a collective reflection on how the new skills and abilities related to the use of new hardware and software tools, were improving each individual performance.

- Activities of collective reflection on how new knowledge and skills of individuals promote better team performance.

- Activities of hands-on, learning by doing of new hardware and software tools.

- Activities with the purpose to coordinate the work within teams that consider the way teams connect existing or previous knowledge or data with other explicit knowledge in their design projects.

Coherence levels grew over each design stage in general for our three design teams, but also was observed an empirical correlation between the growing in coherence in the Socialization Mode and the grow of coherence in the Externalization Mode, but with a slight delay in time. A good example of this is the coherence measures for team A (see Figure 4 and Table 2).

There is a tendency to have cycles of divergent thinking following of convergent process all through the whole new process development as already observed by Agonino [7]. The coherence measurements are expected to grow as the process is passing from a divergent stage to a convergent one.

It would be possible for design teams, to keep a monitoring of their Knowledge Mode Semantic Coherence Level in real time, for the progress in their documents, as well as their web based design activities that are all important potential indicators of a design team's performance within a New Product Development Process.

Acknowledgments. The authors acknowledge the support received by the Instituto Tecnológico y de Estudios Superiores de Monterrey (ITESM) through the Research Chairs in Autotronics. Additional support for this work has been provided by the IBM SUR GRANT. 


\section{References}

1. Wagner, C.: Wiki: A Technology for Conversational Knowledge Management and Group Collaboration. Communication of the Association for Information Systems 13, 265-289 (2004)

2. Nonaka, I.: A Dynamic Theory of Organizational Knowledge Creation. Organization Science 5(1), 14-37 (1994)

3. Landauer, T.K., Foltz, P.W., Laham, D.: Introduction to Latent Semantic Analysis. Discourse Processes 25, 259-284 (1998)

4. Guerra-Zubiaga, D.: A Manufacturing Model to Enable Knowledge Maintenance in Decision Support Systems. Ph.D. Thesis, Loughborough University (2004)

5. Prahalad, C.K., Hamel, G.: The Core Competence of the Corporation. Harvard Business Review 68, 79-91 (1990)

6. http://www.centraldesktop.com

7. Hill, A., Song, S., Dong, A., Agogino, A.M.: A Document Analysis Method for Characterizing Design Team Performance. Journal of Mechanical Design 126(3), 378-385 (2004)

8. Hill, A., Song, S., Dong, A., Agogino, A.M.: Identifying Shared Understanding in Design using Document Analysis. In: Proceedings of the 13th International Conference on Design Theory and Methodology. ASME, Pittsburgh, Pennsylvania (2001)

9. Nonaka, I., Takeuchi, H.: The Knowledge-Creating Company. Oxford University Press, Oxford (1995)

10. http://www.zipfer.com

11. Brohman M.K.: Knowledge Creation Opportunities in the Data Mining Process. In: Proceedings of the 39th Hawaii International Conference on System Sciences. vol. 8, pp. 170c (2006)

12. Guerra-Zubiaga, D., Donato, L., Ramírez, R., Contero, M.: Knowledge Sharing to Support Collaborative Engineering at PLM Environment. In: Reimer, U., Karagiannis, D. (eds.) PAKM 2006. LNCS (LNAI), vol. 4333, pp. 86-96. Springer, Heidelberg (2006)

13. http://lsa.colorado.edu

14. AMR Research, Inc. www.amrresearch.com Source: CSFB/HOLT: Deloitte Consulting Analyses, from The Innovator's Solution, Christiansen and Raynor (2003)

15. Voina, C.: Principles of Semantic Coherence in Concept Learning. In: Proc. of the Singapore Int. Conference. on Intelligent Control and Instrumentation, vol. 2, pp. 736-739 (1992)

16. Guerra, D., Young, R.: A Manufacturing Model to Enable Knowledge Maintenance in Decision Support Systems. In: 33 Annual Conference of North American Manufacturing Research Institution of Society of Manufacturing Engineering (NAMRI/SME), Columbia University, NY, vol. 33-1, pp. 203-210 (2005)

17. Young, R., Cutting-Decelle, A., Guerra, D., Gunendran, G., Das, B., Cochran, S.: Sharing Manufacturing Information and Knowledge in Design Decision Support. In: Bramley, A., Brissaud, D., Coutellier, D., McMahon, C. (eds.) Advanced Integrated Design and Manufacturing in Mechanical Engineering, pp. 173-185. Springer, Heidelberg (2005) 